\title{
Improved TEEN Routing Protocol with Multi Hop and Multi Path in Wireless Sensor Networks
}

\author{
Nilu Kumari \\ M.Tech CSE \\ Department of CSE \\ NIT Jalandhar, India
}

\author{
D.K. Gupta \\ Associate Professor \\ Department of CSE \\ NIT Jalandhar, India
}

\author{
Manoj Kumar Sah \\ Assistant Professor \\ Department of CSE \\ NIT Jalandhar, India
}

\begin{abstract}
Wireless Sensor Networks are used in monitoring environment condition, safety and medical applications. The sensor nodes are usually randomly deployed in a specific region. All these sensor nodes collect their data and send it to the base station (BS) through some routing protocol. These nodes are not rechargeable nodes to keep them alive. These nodes may serve as long as possible without any external assistance, they must follow some protocol that ensure efficient use of their power. In energy consumption of each node a routing technique plays a key role. Many of the routing protocols use clustering as their routing technique. So clustering plays a key role in prolonging the stability period and network life time. The Cluster Heads (CHs) collects data from all the nodes in their cluster, perform aggregation on that and then finally send it to the BS. Sensor nodes must use a certain routing protocol to send their data efficiently to the $\mathrm{BS}$. The objective of all routing protocols is to minimize the energy consumption so that the network lifetime and particularly the stability period of the network may be enhanced. By network lifetime we mean the time duration from the start of the network till the death of the last node whereas, stability period shows the time period from the start of the network till the death of the first node. This paper has an objective to develop an energy efficient increased lifetime threshold sensitive clustering algorithm by dynamic selection of cluster heads using multi-hops and multi-path, that leads to load balancing on different-different clusters. This results in the enhancement of cluster heads or normal nodes network lifetime and comparison of performance of the various proposed clustering protocol with TEEN. In this work we propose an energy efficient multipath routing algorithm in WSN. This protocol is designed to enhance the network life time and energy efficiency through discovering multiple paths from the source to the destination.
\end{abstract}

\section{Keywords}

Wireless Sensor Network, Sink Node, Cluster Head, Routing Protocol, Residual Energy,

\section{INTRODUCTION}

Advances in wireless communication made it possible to develop Wireless Sensor Networks. Wireless Sensor Networks, also abbreviated as WSN, is the new fast growing technology that have been successfully utilized to perform the function of monitoring of the socio-economic areas, environmental conditions, in military applications, in house and many more commercial applications. They consist of spatially distributed sensors which supervise physical or environmental conditions, such as humidity, temperature, sound, pressure. The information collected by these sensors is then passed to the main location through their network by using the interaction among themselves.

These small sensing devices named sensors are also called nodes and consist of a sensing unit, processing unit, power unit and transceiver unit. There are also other sub units, which are application dependent. Most of the routing techniques and sensing tasks require a location finding system to have the knowledge of location with high accuracy in sensor network. Today, wireless sensor networks are widely used in the commercial and industrial areas. The employment of wireless sensor networks is increasing day by day but even after all the advancements it faces the problem of limited battery lifetime. Each node depends on energy for its activities as it alone defines that for how long that node can play an active part in that network, use of energy becomes a major subject to deal within wireless sensor networks. The energy is utilized by the sensing nodes either during the processing of the sensed data or during the communication among themselves or during idle-listening by the nodes.

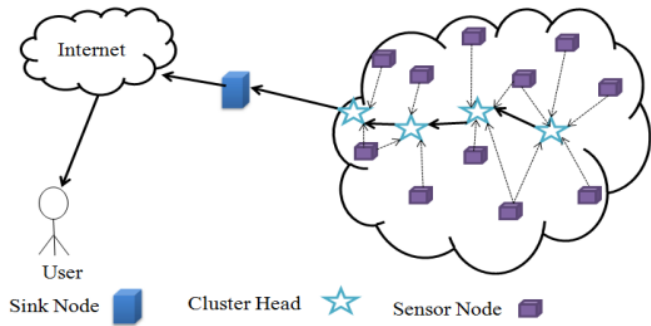

Fig 1: Wireless Sensor Networks

\section{RELATED WORKS}

Despite the multitudinous applications of WSNs, these networks have various limitations, e.g., limited energy supply, and limited bandwidth available to each sensing node for communication etc. It has been noticed that one of the main design goals of WSNs has been to carry out data communication while trying to sustain the nodes for a longer period and also to prevent the connectivity abjection by employing aggressive energy management techniques. Routing protocols design in WSNs hence faces many challenging factors.

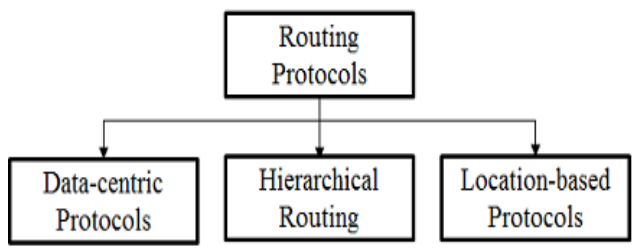

Fig 2: Classifications of Routing Protocols 


\subsection{Data - Centric Protocols}

These were the first set of protocols defined which helps in reducing redundancy. In data-centric routing, the sink or the base station sends queries to certain region nodes in a network and awaits the data from the sensor nodes in the selected regions where event occurred. Hence, if this protocol is used then data is requested through queries. Many data-centric protocols have been proposed so far such as SPIN, Directed Diffusion, Rumor Routing, COUGAR, Acquire and EAD but only some have been described below.

\subsubsection{Sensor Protocols for Information via Negotiation (SPIN)}

It was the first data-centric protocol which was proposed to eliminate redundant data by taking the advantage of the data negotiation between the nodes which thereafter helped reduce the energy consumption. These protocols make use of the property that nodes in propinquity have alike data, and hence there is a need to distribute only the data that other nodes do not possess. It includes a process of exchange of three messages
a. ADV message
b. REQ message
c. DATA message

\subsubsection{Directed Diffusion}

Later another data-centric protocol, Directed Diffusion [5] was developed which become a breakthrough in data-centric routing. This was because thereafter many other protocols were proposed which either were based on Directed Diffusion or followed the similar concept. The idea behind this protocol was to eliminate redundancy by diffusing data coming from different sensor nodes which also helped in the minimization of the number of transmissions, hence saving the energy and prolonging the network lifetime. This protocol consists of three phases

\section{a. Interest Propagation \\ b. Initial Gradients Setup \\ c. Data Delivery}

\subsubsection{Energy Aware Routing}

This data-centric protocol was proposed to provide a set of optimal paths rather than just one optimal path. This set of paths is chosen by means of probability whose value depends upon the energy consumption of each path. This protocol consists of three phases
a. Setup Phase
b. Data Communication Phase
c. Route Maintenance Phase

\subsubsection{COUGAR}

Another data-centric protocol called COUGAR [7] views the network as a huge distributed database system. The main idea is to use declarative queries in order to abstract query processing from the network layer functions such as selection of relevant sensors and so on. COUGAR utilizes in-network data aggregation to obtain more energy savings.

\subsection{Location-based Protocols}

In this kind of routing, sensor nodes are addressed by means of their locations. By using the location information the distance between neighboring nodes can be estimated, thus helping in estimating the amount of energy consumption by the sensor nodes. In these protocols, the location information is utilized in routing data instead of the addressing scheme for sensor networks like IP-addresses. Relative coordinates of neighboring nodes can be obtained by exchanging such information between neighbors. Alternatively, the position of nodes may be available directly by communicating with a satellite using GPS, if nodes are consists with a small low power GPS receiver. To reduce energy consumption, some location based schemes demand that nodes should go to sleep if there is no activity. Energy savings can be increased by having as many sleeping nodes in the network as possible. Some of the location-based protocols are MECN, SMECN, GAF, GEAR, Span, TBF, BVGF, GeRaF.

\subsection{Hierarchical Protocols}

A single-tier network is not scalable for a larger set of sensors as it caused the gateway to overload. As a result, the concept of hierarchical routing was introduced. It allowed energyefficient routing in the networks. The hierarchical architecture basically meant to form a two-layer network where the one layer consisted of the cluster head and the other layer consisted of the similar sensor nodes but they were considered to have lower energy as compared to that of the cluster head. The second layer basically involved routing. The higher energy nodes are used to process and send the information. The creation of clusters and assigning special tasks to cluster heads greatly contributes to the overall system's scalability, lifetime, and energy efficiency. There are many hierarchical protocols LEACH, PEGASIS, HEED, TEEN and APTEEN.

\subsubsection{Low Energy Adaptive Clustering Hierarchy LEACH}

protocol is one of the most popular hierarchical routing algorithms for sensor networks. The abstract idea is to form clusters of the sensor nodes based on the received strength of the signal and use local cluster heads as routers to the BS. This will leads to enhancement of energy since the transmissions done through such cluster heads rather than all sensor nodes. For number of cluster heads there is estimation of 10 percent of the total number of nodes in the networks. For each activity in the $\mathrm{CHs}$ such as data fusion and aggregation are performed locally into the cluster. Cluster heads are not fixed rather it changes randomly over time in order to balance the energy dissipation of nodes. For deciding which node is cluster head depends upon network nodes, choosing a random value that exists between 0 and 1 ? Node becomes a cluster head for the current round if the value is less than the following threshold:

$$
\begin{aligned}
T(n) & =\frac{P}{1-(P *(r \bmod 1 / P))}, \text { ifn } G G \\
& =0, \text { Otherwise }
\end{aligned}
$$

Where, $\mathrm{p}$ is the desired percentage of cluster heads (e.g. 0.1), $\mathrm{r}$ is the current round and $\mathrm{G}$ is the set of nodes that have not been cluster heads in the last $1 / p$ rounds.

\subsubsection{Power-Efficient Gathering in Sensor Information Systems (PEGASIS)}

PEGASIS [2] is the extension of LEACH protocol, which forms chains of sensor nodes so that each nodes transmits and receives from neighboring nodes and only one node from the chain is responsible to transmit data to the sink. The data moves from one node to other node, aggregation performed and transmitted to sink. The formation is done in greedy way. 


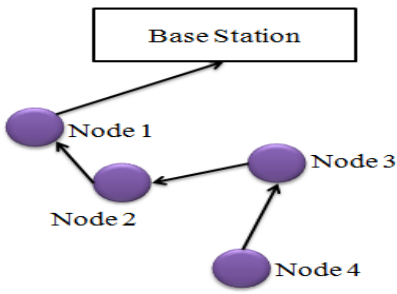

Fig 3 : Flows of Data in Chain to BS

Unlike LEACH, PEGASIS not form clusters and uses only one node from the chain to transmit to sink instead of using many nodes. A sensor transmits data to neighbors in data fusion phase. In PEGASIS, the construction phase supposes that all nodes have global information about the network, especially positions of sensors, and use a greedy approach. When a node dies due to low power, the chain is constructed using same greedy method by bypassing the failed sensor. For every round, a randomly selected node from the chain will transmit aggregated data to sink, thus helps in reduction of per round energy consumption compare to LEACH.

\subsubsection{Hybrid Energy-Efficient Distributed \\ Clustering (HEED)}

HEED is extension of basic principle of LEACH by using residual energy and node degree or density in election of cluster to achieve power balancing.

1. By distributing energy consumption for enhancing network lifetime

2. Termination of clustering process within a fixed number of iterations

3. Reduces control overhead

4. Results in compact and well distributed cluster heads.

In HEED, CHs selection done periodically by considering two clustering parameters. Primary parameter is residual energy of each node and secondary parameter is inert-cluster communication cost. Primary parameter helps in probabilistically selection of an initial set of $\mathrm{CHs}$ and secondary parameter helps in breaking ties. In HEED, a percentage of $\mathrm{CHs}$ among the nodes, $\mathrm{C}_{\text {prob }}$ is the set to assume that an optimal cannot be computed a priori. Probability of each node to become a $\mathrm{CH}$ is:

$$
C H_{\text {prob }}=C_{\text {prob }} \frac{E_{\text {residual }}}{E_{\max }}
$$

Where $E_{\text {residual }}$ is the estimated current energy of the nodes and $E_{\max }$ is the reference maximum energy.

The HEED improves network lifetime compare to LEACH because LEACH randomly selects $\mathrm{CHs}$ which may results in faster death of some nodes. The final CHs selected in HEED are distributed over the network and cost in communication minimized. But the cluster selection with some parameters only, which may impose constraint on system. This helps in prolonging lifetime rather entire needs.

\subsubsection{Threshold Sensitive Energy Efficient Network (TEEN)}

TEEN [3] is also one of the hierarchical protocols. All the nodes report their sensed data to their sensor. The $\mathrm{CH}$ sends the aggregated information to higher level of $\mathrm{CH}$ until the information reaches to the sink. Thus, the architecture of TEEN is based upon the hierarchical grouping where closer nodes form clusters and this process goes on second level until the sink is reached. It uses data-centric technique with hierarchical policy. TEEN is suitable for time critical sensing applications. Also message transmission takes more power than data sensing, so that energy consumption in TEEN protocol is less than the hierarchical protocol in proactive networks. However it is not suitable applications where periodic reports needed because user may not get any data at all if thresholds are not reached.

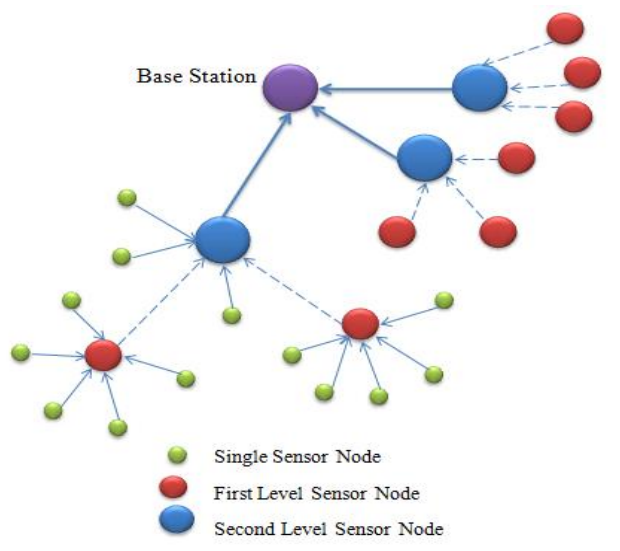

Fig 4 : Clustering Topology in TEEN

\subsubsection{Adaptive Periodic Threshold Sensitive Energy Efficient Sensor Network Protocol (APTEEN)}

APTEEN [4] is an advancement of TEEN protocol to overcome its shortcomings and aims for capturing both periodic as well as time critical data. Thus, it is a hybrid protocol that allows nodes to send their sensed data periodically and react to any sudden change in the value sensed value. The architecture of APTEEN is same as TEEN protocol, which uses the concept of hierarchical energy efficient communication nodes and the sink. APTEEN supports mainly three types of query:
a. One-time query
b. Historical query and
c. Persistent query

These queries are used for monitoring an event for a period of time. APTEEN guarantees low energy dissipation and a large number of sensors alive.

\subsubsection{Energy Efficient Grid Based Clustering $(E E G B C)$}

In EEGBC author has proposed Energy Efficient Grid Based Clustering and routing algorithms for WSNs. This paper addressed the hot spot problem and present grid based clustering and routing algorithms by considering the energy efficiency of the WSN. Routing process consists of three phases:

Initialization phase: This phase include partitioning of target area into grids of equal size and locate position of sink. Grids are constructed based on transmission range of sensor node and sink is placed in the middle of the target area. 


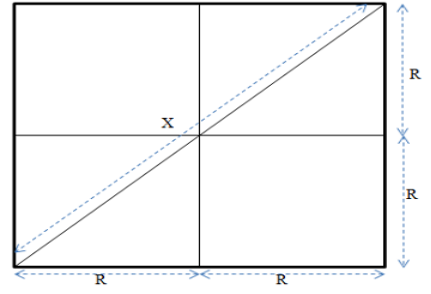

Fig 5: Construction of Grids

Set-up phase: This phase main task is selection of the CHs. Here every grid is a cluster. We calculate the total distance of all the sensor nodes. A node, which is having less distance and residual energy greater than the threshold energy, is designated as $\mathrm{CH}$.

Routing phase: $\mathrm{A} \mathrm{CH} \mathrm{C}_{\mathrm{i}}$ in a particular grid $\mathrm{g}_{\mathrm{i}}$ finds all the $\mathrm{CHs}$ of its neighbor grids and then forwards data to a $\mathrm{CH} \mathrm{C}$ (belonging to a neighbor grid $\mathrm{g}_{\mathrm{i}}$ ), which has least distance from the sink $\mathrm{C}_{\mathrm{j}}$ follows the same procedure for forwarding the data to the next $\mathrm{CH}$ of one of its neighbor grids and the same method is continued until the data finally reached to the sink.

\section{PROPOSED WORK}

Improved TEEN Protocol in Wireless Sensor Networks

A formal classification of wireless sensor networks, on the basis of their way of functioning:

a. Proactive networks

b. Reactive networks.

Reactive networks, as contrary to passive data collecting proactive networks, respond immediately to the changes occurring in the significant parameters of interest. They introduce another energy efficient protocol, TEEN (Threshold sensitive Energy Efficient sensor Network protocol) for reactive networks. The performance of protocol for a simple temperature sensing application was being evaluated. In terms of energy efficiency, the protocol has been observed to outperform existing conventional sensor network protocols. TEEN is based on a hierarchical grouping where closer nodes form clusters and this process goes on the second level until the BS (sink) is reached. TEEN is a clustering communication protocol that targets a reactive network and enables $\mathrm{CHs}$ to impose a constraint on when the sensor should report their sensed data. After clusters are formed, the $\mathrm{CH}$ broadcasts two thresholds to the nodes namely:
a. Hard threshold (HT)
b. Soft threshold (ST).

Hard threshold is the minimum possible value of an attribute, beyond which a sensor should turn its transmitter ON to report its sensed data to its $\mathrm{CH}$. Thus, the hard threshold permits the nodes to transmit data only when the sensed attribute is in the range or domain of interest, thus reducing the number of transmissions significantly. As soon as a node senses a value at or beyond the hard threshold, it transmits data only when the value that attribute changes by an amount equal to or greater than the soft threshold, which indicates a little change in the value of the sensed attribute and triggers a sensor to turn $\mathrm{ON}$ its transmitter and send its sensed data to the $\mathrm{CH}$. As a consequence, soft threshold will further reduce the number transmissions for sensed data if there is little or no change in the value of sensed attribute.
Thus, the sensors will send only sensed data that are of interest to the end user based on the hard threshold value and the change with respect to the previously reported data, thus that yielding more energy savings. One can adjust both hard and soft threshold values in order to control the number of packet transmissions. However, both values of hard soft thresholds have an impact on TEEN. These values should set very carefully to keep the sensors responsive by reporting sensed data to the sink.

However, by using the concept of multi-hop and multi-path on existing TEEN protocol there is some improvement in energy efficiency and network lifetime of the wireless sensor networks.

\subsection{Proposed Algorithm Algorithm}

Step 1: for $\mathrm{i}=1$ to $\mathrm{n}$

$$
\begin{aligned}
& s(i) \cdot x d=r \operatorname{and}(1,1)^{*} x m \\
& X R(i)=s(i) \cdot x d \\
& s(i) \cdot y d=r a n d(1,1)^{*} y m \\
& Y R(i)=s(i) \cdot y d \\
& s(i) \cdot G=0
\end{aligned}
$$

end

Step 2: Creating nodes for multi-hop and multi-path $\mathrm{mm}=$ floor $(\min 1+(\max 1-\min 1) \cdot * \operatorname{rand}(1, \mathrm{n} 11))$

Step 3: After creating a sensor networks we have calculated energy used by the sensor networks

for $\mathrm{I}=1$ to $\mathrm{n}$

if $(\mathrm{d}>\mathrm{do})$

$\mathrm{s}(\mathrm{i}) \cdot \mathrm{E}=\mathrm{s}(\mathrm{i}) \cdot \mathrm{E}-\left((\mathrm{ETx}+\mathrm{EDA}) *(4000)+\mathrm{Emp} * 4000 *\left(\mathrm{~d}^{4}\right)\right)$; else

$s(i) \cdot E=s(i) \cdot E-\left((E T x+E D A) *(4000)+E f s * 4000 *\left(d^{2}\right)\right) ;$

Step 4: Selection of Cluster Head for a cluster

if $\left(\left(\mathrm{s}(\mathrm{i}) \cdot \mathrm{E}==1 \& \&\left(\mathrm{temp} \_\mathrm{rand}<=(\operatorname{padv} /(1-\mathrm{padv} *\right.\right.\right.$ $\bmod (\mathrm{r}, \operatorname{round}(1 / \mathrm{padv})))))))$

Step 5: For detection of dead nodes

for $\mathrm{i}=1$ to $\mathrm{n}$

$$
\begin{aligned}
& \text { if(s2(i). } \mathrm{E}<0) \\
& \text { n_dead=n_dead }+1 \text {; } \\
& \text { else if(s2(i). } E==1 \text { ) } \\
& \text { n_dead_adv }=\text { n_dead_adv }+1 \text {; } \\
& \text { else } \\
& \text { n_dead_nor=n_dead_nor }+1 \text {; }
\end{aligned}
$$

end 


\section{SIMULATION RESULTS}

\subsection{Duration of Network Lifetime}

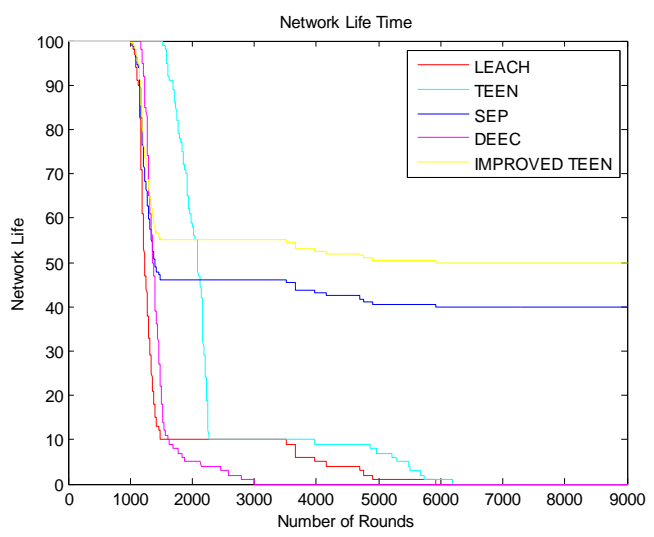

Fig 6: Network Life Time

We note that the IMPROVED TEEN protocol extended significantly the network lifetime compared to others. This improvement with the help of proper distribution of work load by multi-hop and multi-path. Consequently, an energy consumption balancing by the nodes leads to increase in network lifetime.

\subsection{Energy Usage in the Network}

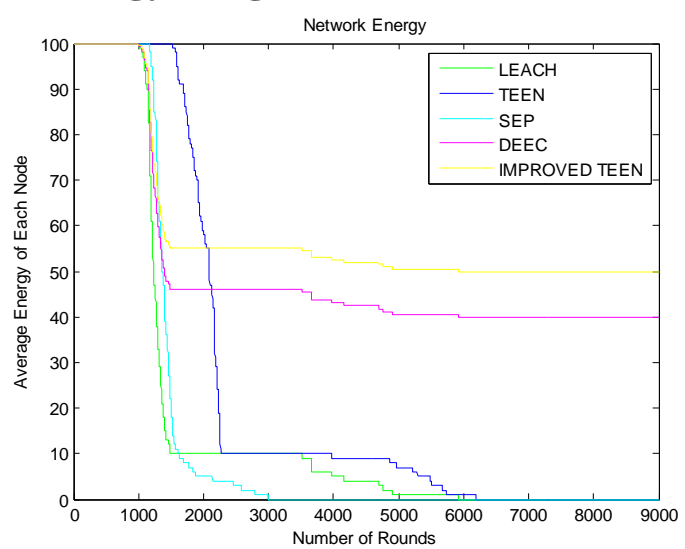

Fig 7 : Total Average Energy of Nodes

\subsection{Number of Nodes Dead}

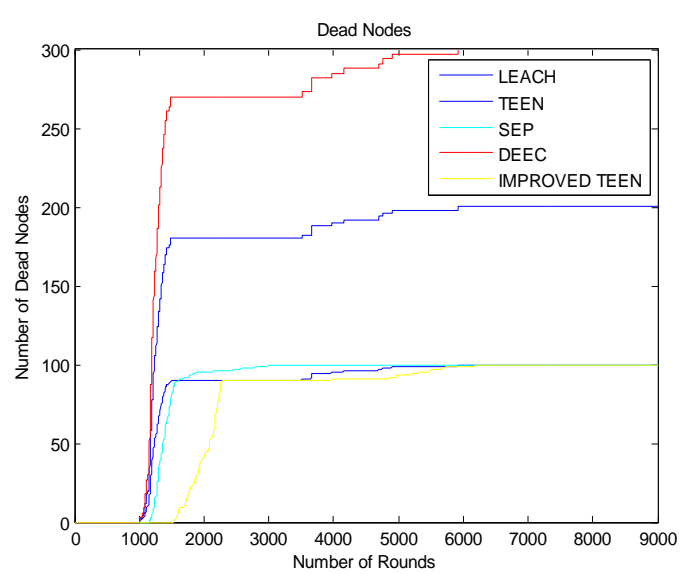

Fig 8: Number of dead Nodes

\section{CONCLUSIONS}

While designing protocol architectures for WSN, it is very important to consider the severe energy constraints of the sensor nodes, the delay of the network, data aggregation and constraints of wireless channel. These features are taken into account in TEEN to some extent, where the amount of data transmitted is reduced by data aggregation at the cluster-head, energy saving is done by introducing multipath and multi-hop in existing WSN. Since each cluster-head in TEEN assigns equal number of slots to all its member nodes, it could lead to delay of the whole network during event driven situations. Various approaches can be adopted to overcome this problem of delay and can be considered as future work.

\section{REFERENCES}

[1] W. Heinzelman, A. Chandrakasan and H. Balakrishnan, "Energy-Efficient Communication Protocol for Wireless Microsensor Networks", Proceedings of the 33rd Hawaii International Conference on System Sciences (HICSS '00), January 2000.

[2] S. Lindsey, C. Raghavendra,"PEGASIS: PowerEfficient Gathering in Sensor Information Systems", IEEE Aerospace Conference Proceedings, Vol. 3, 9-16 pp. 1125-1130, 2002.

[3] A. Manjeshwar and D. P. Agarwal, "TEEN: a routing protocol for enhanced efficiency in wireless sensor networks," In 1st International Workshop on Parallel and Distributed Computing Issues in Wireless Networks and Mobile Computing, April 2001.

[4] Anshu Chauhan, D.K. Gupta, Manoj Kumar Sah, "Detection of Packet Dropping Nodes in MANET using DSR Routing Protocol", International Journal of Computer Applications, USA (0975 - 8887) Volume 123 - No.7, Aug 2015.

[5] C. Intanagonwiwat, R. Govindan, D. Estrin, "Directed diffusion: a scalable and robust communication paradigm for sensor networks", Proceedings of the ACM MobiCom, Boston, MA, 2000.

[6] Jamal N. Al-Karaki, and Ahmed E. Kamal, "Routing Techniques in Wireless Sensor Networks: A Survey", Journal of IEEE Wireless Communications, Volume: 11, Issue: 6, pp. 6-28, 2004.

[7] Y. Yao and J. Gehrke, "The cougar approach to innetwork query processing in sensor networks," in SIGMOD Record, September 2002.

[8] Tejaswi, K.Indian Inst. of Technol. Bombay, Mumbai Mehta, P. Bansal, R. Parekh, C. Merchant, S.N. Desai, U.B., "Routing Protocols for Landslide Prediction using Wireless Sensor Networks" 2006.

[9] ZiboudaAliouat, SaadHarous, "An Efficient Clustering Protocol Increasing Wireless Sensor Networks Life Time" 2012.

[10] C. F. Chiasserini, I. Chlamtac, P. Monti, and A. Nucci, "Energy Efficient Design of Wireless Ad Hoc Networks," Proceedings of European Wireless, Feb 2002.

[11] S. Bandyopadhyay and E. J. Coyle, "An Energy Efficient Hierarchical Clustering Algorithm for Wireless Sensor Networks,” IEEE INFOCOM, April 2003. 
[12] R.C. Shah and J.M. Rabaey, "Energy aware routing for low energy ad hoc sensor networks," Proc. IEEE Wireless Comm. and Networking Conf., pp.350-355, March 2002.

[13] Loscri.V, Morabito.G, Marano.S, “A Two-Level Hierarchy for Low-Energy Adaptive Clustering Hierarchy," In Proceedings of the $2^{\text {nd }}$ IEEE Semiannual Vehicular Technology Conference, Dallas, TX, USA, pp. 1809-1813, 25-28, September 2005.

[14] Fan, X., Song, Y. "Improvement on LEACH Protocol of Wireless Sensor Network," In Proceedings of International Conference on Sensor Technologies and Applications, Valencia, Spain, pp. 260-264, 14-20 October 2007.

[15] Mo Xiaoyan, "Study and Design on Cluster Routing Protocols of Wireless Sensors Networks," Ph.D. Dissertation. Zhejiang University, Hangzhou, China, 2006.

[16] H. Heinzelman, W.B. Chandrakasan, A.P. Balakrishnan, "An application-specific protocol architecture for wireless microsensor networks," IEEE Trans. Wirel. Commun. 1, 660-670, 2002

[17] W. Yassein, M.B. Al-zoubi, A. Khamayesh, Y. Mardini, "Improvement on LEACH protocol of wireless sensor network (VLEACH)," Int. J. Digit. Content Technol. 132-136, Appl. 2009.

[18] S. Ran, G. Zhang, H. Gong, "Improving on LEACH protocol of wireless sensor networks using fuzzy logic," J. Inf. Comput. Sci., 767-775, 2010.

[19] L.K Abdulsalam, H.M. Kamel, "W-LEACH: Weighted Low Energy Adaptive Clustering Hierarchy aggregation algorithm for data streams in wireless sensor networks," In Proceedings of IEEE International Conference on Data Mining Workshops (ICDMW), Sydney, Australia, pp. 1-8., 14, December 2010.

[20] S. Hong, J. Kook, J. Lee, S. Kwon, D. Yi, "T-LEACH: The method of threshold-based cluster head replacement for wireless sensor networks," Inf. Syst. Front, pp. 513$521,2009$. 